\title{
THE INFLUENCE OF CIGARETTE SMOKE ON THE SELECTED BRONCHOALVEOLAR CELLS IN EXPERIMENT
}

\author{
Marta Hurbánková, Silvia Černá, Milan Beňo, Soňa Wimmerová, Štefánia Moricová \\ Slovak Medical University in Bratislava, Faculty of Public Health, Laboratory of Respiratory Toxicology, Bratislava, Slovak Republic
}

\begin{abstract}
SUMMARY
The occurence of lung diseases (obstructive, malignant) resulting from smoking has an increasing tendency. The lung is the primary organ at risk from the effects of inhaled cigarette smoke and smoking has been implicated as a contributing factor to the causation of various respiratory diseases. The aim of presented work was to find out the subchronic effect of the 6-month exposure to cigarette smoke on the selected inflammatory and cytotoxic parameters of bronchoalveolar lavage in $\mathrm{W}$ rats and thus to contribute to understanding of the mechanism of action of tobacco smoke and/or path mechanism of lung injury developed after cigarette smoking. In special chamber, the animals smoked 8 standard research 1R1 type of cigarettes per day, except Saturdays and Sundays, during 6 months. The daily concentration of total particulate matter (TPM) $/ \mathrm{m}^{3}$ air for two hours per exposure requiring to burn eight cigarettes was $85 \mathrm{mg}$. Animals were sacrificed after the 6-month exposure and bronchoalveolar lavage (BAL) was performed and selected inflammatory and cytotoxic BAL parameters were examined and compared with the control group. Following BAL parameters were investigated: the total cell and alveolar macrophages (AM) count in BAL, the differential cell count (\% of AM, \% of polymorphonuclears - PMN, \% of lymphocytes - Ly), proportion of immature AM, proportion of bi-nucleated cells - BNC, viability, the phagocytic activity of AM, cytokines TNF-a (tumor necrosis factor alpha) and IL-1B (interleukin-1beta). Conclusion: A) The 6-month smoking of eight cigarettes daily significantly changed prevailing number of examined BAL parameters; B) The presence of inflammatory and cytotoxic responses in lung tissue can probably signalize beginning or developing of disease process.
\end{abstract}

Key words: cigarette smoke, bronchoalveolar lavage parameters, inflammatory and cytotoxic bronchoalveolar parameters - cytokines

Address for correspondence: M. Hurbánková, Laboratory of Respiratory Toxicology, Slovak Medical University, Limbová 12, SK-83303 Bratislava, Slovakia. E-mail: marta.hurbankova@szu.sk

\section{INTRODUCTION}

More than 5 million people die every year from smokingrelated diseases. The mentioned diseases have furthermore a very important negative impact on the economy due to the loss caused by waste of labour power and increase of the health care and social care costs. Moreover, the high occurence of diseases caused by smoking inhibits the faster rise of the life expectancy of population.

Cigarette smoke contains an amount of chemicals which are very harmful for the health of smokers. By the International Agency for Research on Cancer (IARC) cigarette smoke is classified as a human carcinogen - group 1, which is very harmful not only for smokers, but for non-smokers who inhale tobacco smoke passively as well (1).

Cigarette smoke is an aerosol composed of a gaseous and particle phases. It is a complex mixture containing oxidants and various toxic substances. Many pollutants originating from environmental and industrial sources such as benzene, formaldehyde, lead or arsenic, also occur in cigarette smoke. Among circa 4,800 substances that have as yet been identified in tobacco smoke, there are 69 recognized carcinogens and a large number of toxic substances, some of them highly reactive $(2,3)$. The lung is the primary organ at risk from the effects of inhaled cigarette smoke and smoking has been implicated as a contributing factor to the causation of various respiratory diseases (lung cancer, chronic obstructive pulmonary diseases - COPD), cancer of the breast, brain, stomach, leukemia, lymphomas, coronary heart and peripheral vascular diseases, inflammation of the arteries, progressive narrowing of the vascular lumen, risk of developing myocardial infarction, etc., (3).

Alveolar macrophages (AM) play a significant role in response to tobacco smoke. In addition to being phagocytes, the AM are also important immuno-regulatory cells involved in the defense mechanisms as well as in the pathogenesis of numerous lung diseases $(4,5)$. They release various cytokines, reactive oxygen intermediates, and other mediators of the inflammatory reaction which are important modulators of cell growth and cell differentiation. Since AM are the first cells which come in contact with inhaled noxious substances, they are preferentially used for examination of the cytotoxicity of cigarette smoke. AM also mediate interactions between other cells (polymorphonuclears, lymphocytes, fibroblasts, etc.). Both differential cell counts (AM, PMN, Ly) and an estimate of total cell number and AM are important when reporting the results of cell analysis based on BAL. Each provides important, unique, and complementary data. None of them separately can give the complete information necessary to assess inflammation and possible lung injury. Cytokines are 
polypeptide mediators of cellular homeostasis that have been implicated in the response to lung damage. They can activate and deactivate cellular functions and are important in remodeling of interstitial matrix after injury. Cytokines TNF- $\alpha$ and IL-1ß are involved in inflammatory response, amplitude regulation, and response duration of these effects $(6,7)$.

The aim of the present work was to find out the sub-chronic effect of cigarette smoke on the selected inflammatory parameters of bronchoalveolar lavage in $\mathrm{W}$ rats and contribute so to understanding of the mechanism of the action of tobacco smoke and/or pathomechanism of lung injury developed after cigarette smoking.

\section{MATERIALS AND METHODS}

The study was conducted with the approval of the Animal Ethics Committee of Institute of Preventive and Clinical Medicine, Bratislava and in accordance with the guidelines of the European Convention for the Protection of Vertebrate Animals Used for Experimental and Other Scientific Purposes. Wistar male rats with average weight 192.2 grams at the beginning of exposure and 374.3 grams at the end of experiment were used. All animals were maintained under non-infectious laboratory conditions at $22+2^{\circ} \mathrm{C}, 45 \%$ relative humidity, under natural light and normal light/dark photoperiodicity, and under air condition using the unit WOLF KG 100 (WOLF - Clima Technic, GmbH, Mainburg, Germany). Commercial diet ST 1(TOP - Dovo, Horné Dubové, Slovak Republic) and tap water were available ad libitum. The period of smoking (8 cigarettes/animal/day, except Saturdays and Sundays) lasted 6 months. Standard research cigarettes of the 1R1 type from Tobacco and Health Research Institute - THRI, Lexington, KY, USA were used in experiment. A whole-body actively ventilated exposure chamber was built applying a smoking machine and pumps (THRI) allowing the animals to breathe diluted main-stream tobacco smoke at the target concentration $85 \mathrm{mg}$ of total particulate matter (TPM) $/ \mathrm{m}^{3}$ air for two hours per daily exposure requiring to burn eight cigarettes. The smoke dosage was controlled daily by weighing Cambridge type glass wool filters (THRI) through which smoke was sucked during the exposure period.

Animals ( 8 per group) were sacrificed after 6 month cigarette inhalation, by i.p. thiopental narcosis $-150 \mathrm{mg} / \mathrm{kg}$ of animal and exsanguinated by dissection of the vena cava caudalis. Bronchoalveolar lavage (BAL) was performed and cells were harvested using a modified method of bronchoalveolar lavage by Myrvick et al. (1961) (8). The trachea was cannulated, and the lungs were washed 5 times with $5 \mathrm{ml}$ of saline solution (in situ).

The following selected inflammatory BAL parameters were investigated: the total cell and AM count, the differential cell count (\% of AM, \% of polymorphonuclears - PMN, \% of lymphocytes LY), proportion of immature AM, proportion of binucleated cells - BNC, viability, the phagocytic activity of AM, and cytokines TNF- $\alpha$ and IL- $1 \beta$.

The bronchoalveolar lavage fluid total cells and total AM count was determined in Bürker's chamber and differential counting of macrophages, polymorphonuclear leukocytes (PMNL), lymphocytes (LY), proportion of bi-nucleated cells (BNC) and immature cells in the BALF (bronchoalveolar lavage fluid) were

performed on May-Grünwald Giemsa-Romanowski stained preparations counting 200 cells. Before determination of the count, the cells were cytocentrifuged on one slide by a Cytospin (Shandon) centrifuge.

The phagocytic activity of AM was investigated by the method of Fornůsek et al. using 2-hydroxyethylmetacrylate particles (MSHP) from fy Neosys Prague (9). Fifty $\mathrm{ml}$ of the particles in PBS (phosphate buffer) were added to $100 \mathrm{ml}$ of the BAL fluid and incubated for $60 \mathrm{~min}$ at $37^{\circ} \mathrm{C}$ and shaken at short intervals. Staining was performed by the May-Grünwald Giemsa-Romanowski method. Cells were considered positive when they phagocytized three or more particles .

Viability of AM to $200 \mu 1$ of $0.25 \%$ erythrosine solution was added to $200 \mu \mathrm{l}$ aliquot of the cell suspension. The number of viable and non-viable cells was counted using the Bürker's chamber. For detailed description of aforementioned methods, please refer to authors' paper (10).

TNF- $\alpha$ and IL-1ß produced by activated AM of BAL, were measured using an enzyme-linked immunoadsorbent assay (ELISA) kits. Supernatants of BAL leukocytes were added to microtiter wells pre-coated with anti-monoclonal antibodies. After incubation time of the samples at $37^{\circ} \mathrm{C}$ and thorough washing of the wells, anti-TNF- $\alpha$ and IL- $1 \beta$ were added to the wells. After the second incubation at $37^{\circ} \mathrm{C}$, excess of conjugated antibodies were removed by washing. Then substrate and stop solutions were added, and colour intensity was measured using an ELISA reader (Dynatec) at $450 \mathrm{~nm}$.

All results were statistically evaluated by Man-Whitney test.

\section{RESULTS AND DISCUSION}

The effect of 6-month exposure to cigarette smoke on BAL parameters in rats describes figs. $1-8$.

Compared with the control group of animals, the following effects of treatment with cigarette smoke (after 6-month inhalation) were observed:

- statistically significant increase of the proportion of LY and PMN, percentage of immature AM as well as levels of TNF- $\alpha$ and IL- $1 ß$ cytokines, and

- statistically significant decrease of viability, phagocytic activity of AM and proportion of AM (from differential cell count) in comparison with the control group

- compared with the control, there were no significant affection of the total count of BAL cells, the total count of AM, and the proportion of binucleated cells in BAL.

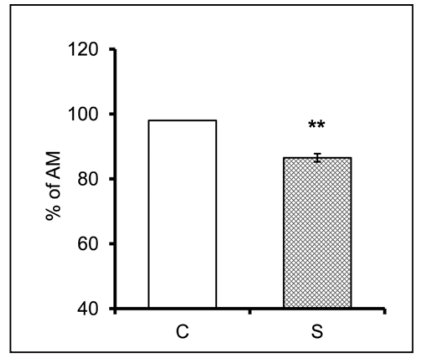

Fig. 1. Proportion of $A M$ in $B A L F$.

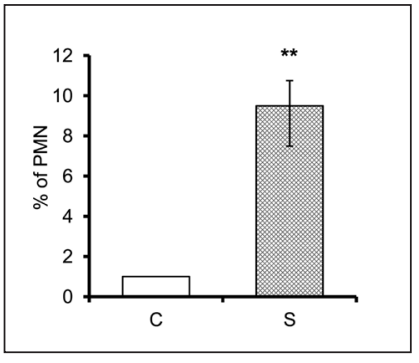

Fig. 2. Proportion of $P M N$ in $B A L F$. 


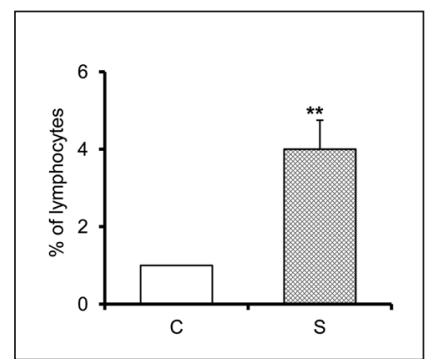

Fig. 3. Proportion of lymphocytes in BALF.

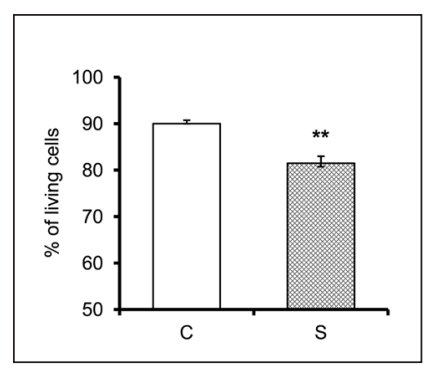

Fig. 5. Viability of AM.

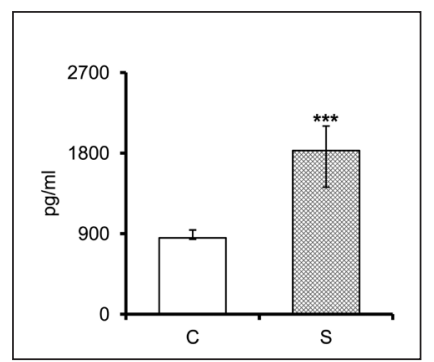

Fig. 7. Level of TNF- $\alpha$ after exposure to cigarette smoke.

$C$ - control group, $S$ - smoking, ${ }^{* *} p<0.01$

The lung is often a major target organ for the toxic effects of many atmospheric pollutants, both gaseous and particulate in nature. The lung responds continually to chemical and physical stimuli. Not all the responses evoked by substances are injurious. Many responses and changes in the lung are not clearly definable as the lung disease or damage, but can be discerned by bronchoalveolar lavage (BAL). In many cases, the cellular constituents obtained in the lavage provide a good indication of lung injury. Examination of the number and type of cells obtained via BAL as well as of their viability and state of activation, enables us to understand the extent of harmful effects caused to the lung by inhaled noxious substances $(3,6)$.

Changes in the lungs due to smoking include inflammation, epithelial damage, and remodeling of the airways. Airway inflammation is likely to play a critical role in the genesis and progression of tobacco smoke-induced airway disease $(3,11)$.

Lung exposure to noxious substances changes the differential cell count by increasing the proportion of inflammatory cells, in particular that of PMN and Ly. However, the reduction in the absolute number of macrophages from the BAL fluid could also account for an increase in the proportion of inflammatory cells $(6,7,12)$. In the present study, we observed that percentage of AM from the differential count was significantly decreased after 6-month inhalation in the rat group (as compared with the corresponding control group). On the other hand, percentage of Ly and PMN was significantly increased (against the controls). In the Kuschneret et al. study, statistically higher concentrations of neutrophils, macrophages, IL-1ß, IL-6, IL- 8 and MCP-1 were observed among smokers compared to nonsmokers $(\mathrm{p} \leq 0.0007$ in all cases) (13). According to Dziedzic et al., long-term recruitment of PMN might be an important factor in prediction of lung metaplastic processes (6).

Blood leukocytosis and increased count of neutrophilles and total inflammatory cells in bronchoalveolar lavage have also been demonstrated in cigarette smokers (14). Chronic obstructive pulmonary diseases (COPD) are also associated with smoking. The lower amount of BAL neutrophils in COPD ex-smokers, compared to COPD smokers, suggests positive alterations in alveolar compartment after smoking cessation $(3,15)$.

AM play a significant role in the mechanism which regulates response to noxious substance exposure. Besides their phagocytic nature, AM are also important immuno-regulatory cells involved in defense mechanisms as well as in the pathogenesis of numerous lung diseases. Activated AM release various cytokines, reactive oxygen intermediates, and other mediators of the inflammatory reaction $(6,7,10,16)$. AM constitute important and frequently utilized tools for examination of cytotoxicity. AM are the predominant cells present in BAL and changes in their number or function are factors determining lung injury and characterizing the pathogenesis of such a response. During the migration of monocytes into tissues, come to a further difference until they become multifunctional tissue macrophages. They may at this stage be regarded as "immature macrophages". During the immune process, the number of "immature macrophages" significantly increases. Decreases in macrophage number, viability or phagocytic capacity may result in impaired clearence of inhaled materials $(6,7,16$, 17). Consequently, our observations of significant decrease in percentage AM and viability as well as significant depression of AM phagocytic activity after cigarette smoke treatment indicate reduction in lung defence and constitute evidence of adverse effects of smoking.

Cigarette smoke and/or many of its components can have direct effects on the secretory and transport functions of the airway epithelial cells. Obviously, such alteration can play an important part in the pathogenesis of chronic airway disease - for example, decreased mucociliary clearence can result in airway obstruction and increased susceptibility to microbial infection. Cigarette smoke can have a number of deleterious effects on intercellular communication and epithelial permeability. These alterations can play a part in the development of chronic respiratory diseases (17, 19). Perturbations of the airway epithelial cell barrier induced by cigarette smoke may lead to adverse changes resulting in airway inflammation. Specific mechanisms involved in this inflammation include: a) increased synthesis and/or release of inflammatory mediators, decreased synthesis of protective mediators; b) synthesis of pro-inflammatory cytokines; c) modulation of cell adhesion molecules; d) modulation of immuno-regulatory processes. Cigarette smoke induces both acute and chronic inflammatory reaction in the airways and lung parenchyma $(3,20)$. 
Oxidant stress placed on lung tissue in smokers may yield superoxide anion which can then be converted to hydrogen peroxide. In the presence of iron, a constituent of tobacco smoke, the Haber-Weiss reaction may then result in formation of hydroxyl radicals that can cause DNA strand breaks. The release of hydrogen peroxide by activated macrophages and lipid peroxidation through reactive aldehydes may place additional oxidant stress in the lung and thus, eventually, result in malignant transformation of the epithelia lining the airways. Inhalation of tobacco smoke decreases the antioxidant defence $(3,21)$. Another potential cause of the smoke-induced airway obstruction could be the development of airway inflammation and associated increases in release of cytokines (IL-1 and TNF- $\alpha$ ) (22).

Cytokines are polypeptide mediators of cellular homeostasis that have been implicated in response to lung damage. They can activate and deactivate cellular functions and are important in remodeling of interstitial matrix after injury. Activated AM release IL-1 and this cytokine influences lymphocytes to produce IFN-gamma which in turn activates AM again. Chemotactic factor (which is also released from AM) attracts granulocytes to foreign substance deposition. ROI from granulocytes are produced (originated) under the influence of TNF-alpha. ROI cause lung tissue injury.

TNF- $\alpha$ play an essential role in lung defence against inhaled noxious substances. In subjects exposed to cigarette smoke in our experiment, significantly increased production of TNF- $\alpha$ and IL-1ß was found. Macrophages may be additionally stimulated to release these factors by production of IFN $-\gamma$ originating from active lymphocytes. TNF- $\alpha$ stimulates leukocytes to production of reactive oxygen intermediates which can impair the tissue injury $(5,16,17)$.

Our results suggested:

- The six month smoking of eight cigarettes daily very significantly changed the majority of examined BAL parameters in rats.

- The presence of inflammatory cells and significant changes in lung defence cells may signalize the process of disease development.

- The long-term inflammation may play an important role in the induction of genotoxicity and carcinogenesis by smoking.

Examined BAL parameters in our study, which were significantly changed in comparison with the control group, are probably involved in the pathomechanism of lung damage. They indicate the cytotoxic effect of cigarette smoke and the ongoing inflammatory processes in lung tissue.

\section{Acknowledgements:}

The work was supported by the grant APVT-21-011104 and MZ SR 2005/29-SZU-07. We wish to thank Ms. H. Bobeková for skilfull technical assistance.
3. Witschi H. Tobacco smoking. In: Gardner DE, editor. Toxicology of the lung. Boca Raton, Fl: CRC/Taylor \& Francis; 2006. p. 623-47.

4. Polosukhin VV, Manouilova LS, Romberger DJ, Matthews KI, Pirruccello SJ, West W, et al. Ultrastructural heterogeneity of the alveolar macrophages from tobacco smokers with chronic bronchitis. Ultrastruct Pathol. 2001 Jan-Feb;25(1):5-11.

5. Stone V, Barlow PG, Hutchison GR, Brown DM. Proinflammatory effect of particles on macrophages and epithelial cells. In: Donaldson K, Borm P, editors. Particle toxicology. Boca Raton, Fl: CRC/Taylor \& Francis; 2007. p. $183-96$.

6. Dziedzic D, Wheeler CS, Gross KB. Bronchoalveolar lavage: detecting markers of lung injury. In: Corn M, editor. Handbook of hazardous materials. San Diego: Academic Press; 1993. p. 99-111.

7. Hurbánková M, Kaiglová A, Buchancová J. Cytokines - the important biomarkers of lung injury after exposure to industrial fibrous dusts. Acta Medica Martinina. 2001;(1):9-24.

8. Myrvik Q, Leake ES, Fariss B. Studies on pulmonary alveolar macrophages from the normal rabbit: a technique to procure them in a high state of purity. J Immunol. 1961 Feb;86:128-32.

9. Fornusek L, Větvička V, Kopeček J. Phagocytosis of the peritoneal leukocytes a new single method. Immunol Zprav. 1982;(13):67-8. (In Czech.)

10. Hurbánková M, Kaiglová A. Compared effects of asbestos and wollastonite fibrous dusts on various biological parameters measured in bronchoalveolar lavage fluid. Journal of Trace and Microprobe Techniques. 1999;(17):233-43.

11. Smith KR, Pinkerton KE, Watanabe T, Pedersen TL, Ma SJ, Hammock $\mathrm{BD}$. Attenuation of tobacco smoke-induced lung inflammation by treatment with a soluble epoxide hydrolase inhibitor. Proc Natl Acad Sci U S A. 2005 Feb 8;102(6):2186-91.

12. Pittet JF, Mackersie RC, Martin TR, Matthay MA. Biological markers of acute lung injury: prognostic and pathogenetic significance. Am J Respir Crit Care Med. 1997 Apr;155(4):1187-205.

13. Kuschner WG, D'Alessandro A, Wong H, Blanc PD. Dose-dependent cigarette smoking-related inflammatory responses in healthy adults. Eur Respir J. 1996 Oct;9(10):1989-94.

14. Anderson R, Theron AJ, Richards GA, Myer MS, van Rensburg AJ. Passive smoking by humans sensitizes circulating neutrophils. Am Rev Respir Dis. 1991 Sep;144(3 Pt 1):570-4.

15. Babusyte A, Stravinskaite K, Jeroch J, Lötvall J, Sakalauskas R, Sitkauskiene B. Patterns of airway inflammation and MMP-12 expression in smokers and ex-smokers with COPD. Respir Res. 2007 Nov 14;8:81.

16. Tarkowski M, Górski P. Macrophage activity in asbestos related diseases. Pol J Occup Med Environ Health. 1991;4(2):115-25.

17. Cohen MD. Pulmonary immunotoxicology. In: Gardner DE, editor Toxicology of the lung. Boca Raton, Fl: CRC, Taylor \& Francis; 2006. p. 351-418.

18. Hurbánková M, Tátrai E, Černá S, Six É, Kováčiková Z, Kyrtopoulos $\mathrm{S}$. Inflammatory and cytotoxic effects as well as histological findings of selected industrial fibrous dusts in Fischer rats after intratracheal instillation. Biologia. 2004;59(6):761-78.

19. Dye JA, Adler KB. Effects of cigarette smoke on epithelial cells of the respiratory tract. Thorax. 1994 Aug;49(8):825-34.

20. Sparrow D, Glynn RJ, Cohen M, Weiss ST. The relationship of the peripheral leukocyte count and cigarette smoking to pulmonary function among adult men. Chest. 1984 Sep;86(3):383-6.

21. Frank JK, Mudway IS. Particle-mediated extracellular oxidative stress in the lung. In: Donaldson K, Borm P, editors. Particle Toxicology. Boca Raton, Fl: CRC/Taylor \& Francis; 2007. p. 89-118.

22. Endo T, Uchida Y, Matsumoto H, Suzuki N, Nomura A, Hirata F, et al. Regulation of endothelin-1 synthesis in cultured guinea pig airway epithelial cells by various cytokines. Biochem Biophys Res Commun. 1992 Aug 14;186(3):1594-9.

\section{REFERENCES}

1. IARC Working Group on the Evaluation of Carcinogenic Risks to Humans. Tobacco smoke and involuntary smoking. IARC Monogr Eval Carcinog Risks Hum. 2004;83:1-1438.

2. Aufderheide M. Artificially generated cigarette smoke - a model for studying komplex atmospheres. In: Dillitzer A, Straudi A, editors. Fraunhofer ITEM news report. 2005. p. 1-2. 